\title{
Leucovorin/Fluorouracil/Liposomal Irinotecan Regimen
}

National Cancer Institute

\section{Source}

National Cancer Institute. Leucovorin/Fluorouracil/Liposomal Irinotecan Regimen. NCI

Thesaurus. Code C158077.

A chemotherapy regimen consisting of folinic acid (leucovorin), 5-fluorouracil, and liposomal irinotecan that may be used in the treatment of adenocarcinoma of the pancreas. 